\title{
Evidências da relação entre participação orçamentária, ambiguidade de papéis e o desempenho gerencial*
}

\author{
Evidencias de la relación entre participación presupuestaria, ambigüedad de papeles y el desempeño gerencial
}

Evidence of the Relationship Between Budgetary Participation, Role Ambiguity and Managerial Performance

\author{
Andréia Acássia Guidini ${ }^{\text {a }}$ \\ Universidade do Contestado UnC, Brasil \\ guidiniandreia@hotmail.com \\ ORCID: http://orcid.org/0000-0001-7919-620X \\ Vinícius Costa da Silva Zonatto \\ Universidade Federal de Santa Maria, Brasil \\ ORCID: http://orcid.org/0000-0003-0823-6774

\section{Larissa Degenhart} \\ Universidade Federal de Santa Maria, Brasil \\ ORCID: http://orcid.org/0000-0003-0651-8540 \\ Daiani Schlup \\ Universidade Regional de Blumenau, Brasil \\ ORCID: http://orcid.org/0000-0002-8052-6250
}

DOI: https://doi.org/10.11144/Javeriana.cc21.erpo

Recepção: 17/05/2019

Aprovação: 13/02/2020

Publicação: 05/06/2020

\section{Resumo:}

Esta pesquisa analisa os efeitos da ambiguidade de papéis na relação entre a participação orçamentária e o desempenho gerencial. A amostra foi composta por 150 gestores com responsabilidades orçamentárias das maiores empresas brasileiras exportadoras. Utilizou-se a técnica de análise de trajetórias. Os resultados indicaram que os gestores com responsabilidades orçamentárias de empresas brasileiras percebem a ambiguidade de papéis quando desempenham suas tarefas no contexto orçamentário. Além disso, a participação dos gestores na definição de metas e objetivos orçamentários e o envolvimento destes no estabelecimento do orçamento das suas unidades possibilitou minimizar a ambiguidade de papéis dos gestores pesquisados, e consequentemente melhorou o desempenho gerencial destes no que tange a um maior êxito na realização das atividades orçamentárias. Conclui-se que a participação orçamentária é um importante constructo que deve ser considerado nas organizações, visto que auxilia para a constante troca de informaçóes sobre os deveres a serem cumpridos, as responsabilidades do cargo dos gestores e, ainda, o comportamento esperado no desempenho das tarefas, funções no contexto orçamentário.

\section{Código JEL: M41}

Palavras-chave: participação orçamentária, ambiguidade de papéis, desempenho gerencial.

\section{Resumen:}

Esta investigación analiza los efectos de la ambigüedad de roles en la relación entre la participación presupuestal y el desempeño gerencial. La muestra fue compuesta por 150 gerentes con responsabilidades presupuestarias de las mayores empresas brasileñas exportadoras. Se utilizó la técnica de análisis de trayectorias. Los resultados indicaron que los gerentes con responsabilidades presupuestarias de empresas brasileñas perciben la ambigüedad de roles cuando desempeñan sus tareas en el contexto presupuestal. Además, la participación de los gerentes en la definición de metas y objetivos presupuestales y su intervención en la asignación del presupuesto de sus unidades hizo posible minimizar la ambigüedad de roles de los gerentes encuestados y, en consecuencia, mejoró su desempeño gerencial en lo que se refiere a un mayor éxito en la realización de las actividades presupuestales. Se concluye que la participación presupuestal es un constructo importante que debe ser considerado en las organizaciones, ya que auxilia en el constante intercambio de informaciones sobre los deberes a ser cumplidos, las responsabilidades del cargo de los gerentes e incluso el comportamiento esperado frente al desempeño de las tareas, funciones en el contexto presupuestal.

Código JEL: M41

\section{Autor notes}

${ }^{a}$ Autora para correspondência. Correio eletrônico: guidiniandreia@hotmail.com 
Palabras clave: participación presupuestal, ambigüedad de papeles, rendimiento gerencial.

\section{Abstract:}

This research analyzes the effects of role ambiguity on the relationship between budget participation and managerial performance. The sample was composed of 150 managers with budgetary responsibilities of the largest Brazilian exporting companies. The trajectory analysis technique used. The results indicated that managers with budgetary responsibilities of Brazilian companies perceive the ambiguity of roles when they perform their tasks in the budget context. In addition, the participation of managers in the definition of budget goals and objectives and their involvement in establishing the budget of their units made it possible to minimize the role ambiguity of the surveyed managers, and consequently improved their managerial performance in terms of greater success in budgetary activities. It is concluded that budget participation is an important construct that must be considered in organizations, since it assists in the constant exchange of information about the duties to be fulfilled, the responsibilities of the manager's position and, also, the expected behavior in the performance of tasks, functions in the budget context.

JEL Code: M41

Keywords: Budgetary participation, role ambiguity, management performance.

\section{Introdução}

O orçamento é um dos tópicos amplamente pesquisados na literatura sobre contabilidade gerencial e tem sido pesquisado a partir de perspectivas teóricas de três diferentes áreas do conhecimento: economia, psicologia e sociologia (Shields \& Shields, 1998; Covaleski et al., 2003; 2007). Este estudo pauta-se na perspectiva psicológica. A pesquisa orçamentária participativa baseada em teorias psicológicas considera três mecanismos por meio dos quais a participação orçamentária promove o envolvimento entre superior e seus subordinados: o efeito cognitivo, do valor de realização e motivacional.

O efeito cognitivo, foco deste estudo, pressupõe que a participação orçamentária melhora o desempenho do subordinado, aumentando a qualidade da tomada das decisões, como resultado do compartilhamento de informações entre superior e seus subordinados (Shields \& Shields, 1998). De acordo com Milani (1975) a participação orçamentária refere até que ponto um subordinado pode selecionar seus cursos de ação na organização. É um processo pelo qual os subordinados possuem a oportunidade de se envolver e influenciar o processo de estabelecimento do orçamento (Parker \& Kyj, 2006), sendo um importante tema de estudo na contabilidade gerencial (Birnberg, Luft, \& Shields, 2007).

A perspectiva da psicologia pode ser utilizada para explicar as causas e os efeitos das práticas contábeis na mente e no comportamento dos indivíduos (Birnberg et al., 2007; Frezatti et al., 2010). A contabilidade, ao lidar com os processos de tomada de decisão, utiliza-se de princípios comportamentais da psicologia (Covaleski et al., 2007; Birnberg et al., 2007; Lucena, Fernandes \& Silva, 2011).

No que tange aos efeitos cognitivos, dois conceitos chave na teoria das funções que estão relacionados à pesquisa contábil gerencial são o conflito e ambiguidade de papéis (Birnberg et al., 2007). Estes dois tipos de tensões organizacionais podem ser originados de fatores organizacionais, pessoais e interpessoais que afetam as percepções dos executivos enquanto cumprem suas responsabilidades (Fisher, 2001; Fichter, 2011). A ambiguidade de papéis ocorre quando os indivíduos não possuem clareza das suas tarefas, dos melhores métodos para atender as expectativas da função, as consequências de diversos aspectos relacionados ao desempenho da função e as expectativas dos superiores em relação as tarefas (Lin \& Ling, 2018).

O papel cognitivo da participação orçamentária sugere que a participação dos subordinados nos processos orçamentários lhes proporciona oportunidades de reunir, trocar e disseminar informações relevantes para a realização do trabalho, o que facilita sua tomada de decisão e melhora o seu desempenho no trabalho (Chenhall \& Brownell, 1988; Chong, 2002; Chong \& Chong, 2002; Zonatto, Weber \& Nascimento, 2019; Jacomossi, Schlup \& Zonatto, 2019).

Chong, Eggleton e Leong (2006), Zonatto et al. (2019) e Jacomossi et al. (2019) propuseram que o efeito cognitivo da participação orçamentária reduz a ambiguidade de papéis. Nesta pesquisa, também 
propomos que a participação orçamentária minimiza a ambiguidade de papéis dos subordinados, ao fornecelhes informações claras sobre as tarefas e métodos para cumprir suas expectativas, o que pode conduzir a que apresentem melhor desempenho no trabalho. Esta lacuna teórica dos efeitos cognitivos da participação orçamentária no desempenho gerencial demanda de maior atenção por parte dos pesquisadores no cenário brasileiro. Este gap de pesquisa permanece na área comportamental da contabilidade, constituindo-se uma oportunidade de pesquisa (Dani, Zonatto \& Diehl, 2017).

Diante do exposto, esta pesquisa visa sanar a seguinte questão: Quais são os efeitos da ambiguidade de papéis na relação entre a participação orçamentária e o desempenho gerencial? Para tanto, este estudo objetiva analisar a relação entre estas variáveis levando em consideração a ambiguidade de papéis.

Uma contribuição do estudo relaciona-se com a relevância da temática, pois localizaram-se poucos estudos nacionais que utilizaram a ambiguidade de papéis como uma variável mediadora para a análise da relação entre a participação orçamentária e o desempenho gerencial (Zonatto et al., 2019; Jacomossi et al., 2019). Considerando que durante o processo orçamentário, os gestores passam por tensões relacionadas à ambiguidade de papéis e isso pode afetar o seu desempenho no trabalho (Palomino \& Frezatti, 2016), denotase relevante interpretar a ambiguidade de papéis no cenário brasileiro, visto que pode apresentar um impacto negativo no desempenho.

Justifica-se a realização desta pesquisa por apresentar uma possível explicação para os resultados conflitantes encontrados na literatura entre a participação orçamentária e o desempenho gerencial (Zonatto \& Lavarda, 2013; Jermias \& Yigit, 2013; Zonatto, 2014; Santos, Lavarda \& Marcello, 2014; Almasi, Palizdar \& Parsian, 2015), sugerindo que a participação orçamentária pode também influenciar indiretamente o desempenho gerencial. Neste estudo, por meio da variável ambiguidade de papéis, busca-se revelar que a mesma pode potencializar a relação entre a participação orçamentária e o desempenho gerencial. Este estudo também revelará que a pesquisa comportamental na contabilidade é incipiente ao observar o efeito de poucas variáveis em sua investigação, constituindo-se dessa forma um campo de pesquisa ainda pouco explorado (Dani et al., 2017).

Destaca-se a necessidade de analisar a temática proposta, pois Palomino e Frezatti (2016) abordam que as situações que poderiam incentivar a ambiguidade de papéis nos gestores com responsabilidades orçamentárias relacionam-se com a complexidade enfrentada na contemporaneidade pelas empresas brasileiras, que afeta diretamente a área de controle. Justifica-se ainda pela importância do entendimento do potencial dos orçamentos no nível do indivíduo, importância evidente nas pesquisas sobre atitudes e comportamentos dos indivíduos, sendo a ambiguidade de papéis uma variável que fornece a base para a análise da funcionalidade potencial dos orçamentos no nível do indivíduo (Marginson \& Ogden, 2005; Zonatto et al., 2019; Jacomossi et al., 2019).

Assim, espera-se que em condições de maiores níveis de participação orçamentária, ocorram efeitos cognitivos positivos sobre o desempenho gerencial, mediante a redução da ambiguidade de papéis. De acordo com Zonatto (2014), este estudo auxilia para o desenvolvimento de pesquisas futuras voltadas para a área comportamental da contabilidade sob a perspectiva gerencial, temática pouco analisada no Brasil, na medida em que os achados revelam importantes questóes em aberto na literatura contábil que podem ser abordadas com o desenvolvimento de novas pesquisas.

\section{Fundamentação teórica e hipóteses da pesquisa}

\section{Participação orçamentária e desempenho gerencial}

A teoria da psicologia sugere que a participação orçamentária influencia positivamente o desempenho dos indivíduos no trabalho. No entanto, as investigações sobre a participação do indivíduo neste processo 
têm revelado resultados inconsistentes (Chong \& Jonhson, 2007; Dani et al., 2017). Conforme Shields e Shields (1998), no processo de participação orçamentária o gestor é envolvido na elaboração e possui influência na definição do orçamento da sua unidade, pois há o compartilhamento de informações entre superior e seus subordinados e coordenação de interdependências, o que possibilita motivar e promover atitudes e comportamentos positivos nos indivíduos no trabalho. Assim, compartilhar as metas e objetivos orçamentários poderá facilitar a alocação de recursos, de modo que sejam suficientes para o desenvolvimento das atividades na unidade sob responsabilidade do gestor (Zonatto et al., 2019), logo, a participação orçamentária tende a favorecer o desempenho deste no trabalho.

Macinati, Bozzi e Rizzo (2016) ressaltam que as empresas devem buscar o envolvimento dos gestores no processo orçamentário, pois os recursos e práticas organizacionais apoiam o comportamento dos gestores e apresentam reflexos no seu desempenho gerencial. Denota-se que o estilo orçamentário (participativo ou não participativo) deve ser congruente com as atitudes e motivações dos gestores, para que se possa obter um desempenho gerencial favorável (Dani et al., 2017).

Contudo, os resultados identificados na literatura são conflitantes, especificamente sobre os efeitos da participação orçamentária no desempenho gerencial, o que estimula a realização de novos estudos (Zonatto, 2014; Derfuss, 2016). Identificaram-se pesquisas que apresentaram resultados positivos entre tais relacionamentos (Zonatto \& Lavarda, 2013; Santos et al., 2014; Almasi et al., 2015; Hariyanti, Purnamasari \& Lestira O, 2015; Zonatto et al., 2019; Jacomossi et al., 2019), negativos (Etemadi et al., 2009), e até mesmo achados que não encontraram relação entre estas variáveis (Chenhall \& Brownell, 1988; Chong et al., 2006; Venkatesh \& Blaskovich, 2012; Jermias \& Yigit, 2013; Macinati et al., 2016).

Zonatto e Lavarda (2013) analisaram as características orçamentárias de uma organização e revelaram que a empresa possui configuração orçamentária participativa e que o orçamento é utilizado para a formação de objetivos, estabelecimento de metas e a avaliação de desempenho. Nesse sentido, o orçamento é elaborado em um determinado período de tempo como uma diretriz na realização das atividades operacionais e como um meio para avaliar o desempenho gerencial (Hariyanti et al., 2015), o que denota que a participação orçamentária tende a elevar o desempenho dos gestores nas suas atividades orçamentárias.

Almasi et al. (2015) abordam algumas recomendações relacionadas ao impacto da participação dos gestores nos processos orçamentários sobre o desempenho gerencial. Dentre as recomendações, destacam-se: melhorar a taxa de participação dos subordinados na elaboração do orçamento; aprimorar os argumentos oferecidos pelos gestores sobre o processo orçamentário, bem como pesquisar sugestóes dos gerentes por parte de seus superiores com vistas a melhorar a participação destes. Portanto, o desempenho gerencial esperado da participação orçamentária é suportado e desafiado.

Apesar de tais resultados, Zonatto (2014) evidenciou que as métricas utilizadas em estudos anteriores para mensurar o desempenho gerencial e avaliar a relação deste com a participação orçamentária pode ter influenciado a identificação de resultados divergentes na literatura. No entanto, vários estudos expuseram um desempenho gerencial efetivo em condições de participação na elaboração do orçamento. Nesta óptica, espera-se que um alto grau de envolvimento e influência na elaboração e definição do orçamento esteja significativamente relacionado a um alto desempenho gerencial, formulando-se a seguinte hipótese: $\mathrm{H}_{1}$ : Existe relação positiva entre a participação orçamentária e o desempenho gerencial.

\section{Participação orçamentária e ambiguidade de papéis}

A segunda hipótese diz respeito ao papel cognitivo da participação orçamentária, sendo este papel avaliado por meio da ambiguidade de papéis, conceituada como a lacuna existente entre as informações necessárias para executar adequadamente um trabalho e as informações disponíveis. Existe ambiguidade de papéis quando o indivíduo tem informações insuficientes para realizar seu trabalho ou quando os deveres, autoridade e responsabilidades não são claros (Burney \& Widener, 2007). 
A ambiguidade de papéis é um estressor relacionado ao trabalho e pode impedir o crescimento individual (Wincent \& Örtqvist, 2011). Fisher (2001) e Palomino (2013) apontam que a ambiguidade de papéis é uma tensão organizacional que pode ser percebida por um indivíduo em decorrência da falta de informação para desempenhar suas funções. Palomino e Frezatti (2016) complementam que a ambiguidade de papéis é considerada a incerteza sobre o que uma pessoa responsável por uma tarefa específica deve fazer.

Segundo a teoria das funções, a ambiguidade de papéis possibilita aumentar a probabilidade de um indivíduo estar insatisfeito com as suas tarefas, e assim, terá ansiedade e irá desenvolver o seu trabalho de forma ineficaz (Rizzo, House e Lirtzman, 1970). Perante isto, o mecanismo cognitivo pressupõe que a participação orçamentária aufere aos subordinados a oportunidade de compartilhar os seus conhecimentos com os seus superiores e permite discussões mais abertas no contexto orçamentário (Chong; 2002; Chong \& Chong, 2002).

No que tange à relação entre a participação orçamentária e a ambiguidade de papéis, estudos empíricos (Chenhall \& Brownell, 1998; Chong \& Bateman, 2000; Chong, 2002; Chong et al., 2006; Parker \& Kyj, 2006; Jermias \& Yigit, 2013; Zonatto et al., 2019) apoiam uma associação negativa entre tais variáveis. Essas pesquisas propuseram que o efeito cognitivo da participação orçamentária minimiza a ambiguidade de papéis dos subordinados (Chenhall \& Brownell, 1988; Chong \& Bateman, 2000; Chong et al., 2006), pois o processo de participação fornece-lhes informações claras sobre o desenvolvimento de suas tarefas e métodos para cumpri-las.

Em relação aos métodos a serem empregados no cumprimento das tarefas, a participação orçamentária proporciona a oportunidade para o gerente trazer conhecimentos locais e especializados de suas tarefas ao processo de elaboração do orçamento, permitindo neste uma discussão mais aberta. Assim, a participação no processo orçamentário proporciona meios para minimizar a ambiguidade de papéis existente no contexto orçamentário (Chenhall \& Brownell, 1988). Por outro lado, a pesquisa desenvolvida por Jacomossi et al. (2019) encontrou resultados de uma relação positiva, achado contrário ao esperado pelos autores, o que revela que a maioria dos gestores analisados percebem a ambiguidade de papéis em suas organizações, e que mesmo havendo maiores níveis de participação orçamentária, há ambiguidade de papéis.

Chong et al. (2006) ressaltam que a participação orçamentária oferece aos subordinados a oportunidade de partilhar, esclarecer e discutir as informações orçamentárias com seus pares e superiores, o que faz com que a disponibilidade de informações auxilie no esclarecimento dos papéis. Jermias e Yigit (2013) salientam que a participação dos subordinados durante o processo orçamentário pode auxiliar no esclarecimento das tarefas que estes deverão realizar.

Alem disso, destaca-se que o orçamento participativo fornece aos subordinados a oportunidade de compartilhar informações com seus superiores (Shields \& Shields, 1998) e essa troca de informações ajudará a esclarecer as expectativas dos papéis dos subordinados, os métodos para cumprir as tarefas e questões voltadas ao desempenho destes no trabalho (Chong et al., 2006). Neste contexto, propomos que o papel cognitivo da participação orçamentária reduz os níveis de ambiguidade de papéis dos subordinados: $\mathbf{H}_{2}$ : Existe relação negativa entre a participação orçamentária e a ambiguidade de papéis.

\section{Ambiguidade de papéis e desempenho gerencial}

A ambiguidade de papéis pode ser considerada uma fonte de obstáculos que impedem as emoções positivas e a realização eficiente das tarefas, o que, por sua vez, pode levar a comportamentos e desempenho no trabalho insatisfatórios (Lin \& Ling, 2018). De acordo com Chenhall e Brownell (1988), níveis elevados de ambiguidade de papéis podem resultar em um declínio no desempenho. A ambiguidade de papéis pode conduzir a um menor desempenho no trabalho, pois elevada ambiguidade indica que um subordinado é incerto sobre o seu papel dentro da organização e isso inclui incerteza sobre as expectativas organizacionais e os meios necessários para cumpri-las (Parker \& Kyj, 2006). 
Situações de ambiguidade de papéis podem levar à incapacidade dos indivíduos de reconhecer os fatores relacionados ao desempenho que o afetam negativamente no trabalho, pois os subordinados estão principalmente envolvidos em esclarecer as expectativas dos papéis e, assim, passando a lidar com os estressores no ambiente de trabalho (Burkert, Fischer \& Schäffer, 2011). Palomino e Frezatti (2016) destacam algumas circunstâncias que podem gerar ambiguidade de papéis nos controllers brasileiros, como por exemplo, falta de informação sobre os deveres a serem cumpridos, responsabilidades e expectativas voltadas ao cargo que ocupam e o comportamento esperado do executivo na sua função como controller.

No contexto orçamentário, as evidências encontradas na literatura têm revelado que a participação dos gestores nos processos orçamentários contribui para a redução da ambiguidade de papéis e melhora o desempenho do indivíduo no trabalho, visto que constataram uma relação negativa entre ambiguidade de papéis e desempenho gerencial (Chenhall \& Brownell, 1988; Tubre \& Collins, 2000; Fischer, 2001; Chong, 2002; Marginson \& Ogden, 2005; Chong et al., 2006; Parker \& Kyj, 2006; Wu \& Norman, 2006; Chong \& Jonhson, 2007; Burney \& Widener, 2007; Gilboa et al., 2008; Jermias \& Yigit, 2013; Zonatto et al., 2019). Burkert et al. (2011), Marginson, McAulay, Roush e Van Zijl (2014), Beuren, Roth e Anzilago (2017) e Beuren, Anzilago e Dal Vesco (2018), analisaram outro cenário, não sendo os efeitos da participação orçamentária e mostraram também que a ambiguidade de papéis se relaciona negativamente com o desempenho gerencial.

Esta relação negativa ocorre a partir do compartilhamento de informações entre superior e subordinado, uma vez que este fato permite o alcance de melhor desempenho (Parker \& Kyj, 2006; Chong \& Jonhson, 2007). Tais resultados indicam ainda que a diminuição da ambiguidade de papéis está associada o melhor desempenho gerencial em termos de planejamento estratégico e tomada de decisão (Burney \& Widener, 2007). Contudo, a pesquisa de Jacomossi et al. (2019) revelou que a ambiguidade de papéis não apresentou efeitos significativos no desempenho gerencial. Evidencia-se, conforme os resultados, que a clareza e a exatidão relacionadas às atividades a serem desempenhadas, possivelmente não estão sendo comunicadas de maneira pertinente aos gestores. Outra justificativa é que em função da complexidade das tarefas, a compreensão dos gestores com responsabilidades orçamentárias esteja sendo prejudicada durante sua participação no processo orçamentário.

Rizzo et al. (1970) argumentam que a elevada ambiguidade de papéis causa menor produtividade, maior tensão no trabalho, insatisfação e retração psicológica. À medida que a complexidade do trabalho aumenta, junto com a maior responsabilidade na tomada de decisões, pode-se esperar maior ambiguidade de papéis no ambiente de trabalho (Tubre \& Collins, 2000). A ambiguidade de papéis pode ser percebida como um desafio nas organizações que buscam facilitar e recompensar o envolvimento dos indivíduos no trabalho. No entanto, é percebida como uma ameaça para os subordinados que visam operações padronizadas e que recompensem o seu desempenho (Gilboa et al., 2008). Burkert et al. (2011) salientam que a ambiguidade de papéis prejudica o desempenho individual.

Nessa ordem de ideias, os gestores desempenham diversos papéis em uma organização, como por exemplo, planejamento, estabelecimento de direções, organização de tarefas, alocação de recursos, liderança, entusiasmo na força de trabalho, e a obtenção dos resultados desejados (Schermerhorn Jr., Hunt, \& Osborn, 2007), o que consequentemente pode minimizar a ambiguidade de papéis dos gestores e melhorar o seu desempenho no trabalho. Por outro lado, a ambiguidade de papéis pode resultar em menores níveis de desempenho, pois representará falta de informações entre superior e subordinados (Tubre \& Collins, 2000).

Entretanto, os gerentes confrontados com incertezas relacionadas com a ambiguidade de papéis, podem reagir positivamente ao se envolver na consecução dos objetivos e metas orçamentárias, posto que a participação orçamentária oferece estrutura e clareza para as tarefas a serem desenvolvidas. A redução da ambiguidade de papéis pode exercer considerável influência no comportamento dos indivíduos no contexto orçamentário, garantindo-lhes maior compromisso com o orçamento da unidade e visando experiências com 
as tarefas (Marginson \& Ogden, 2005). Para tanto, propomos que: $\mathbf{H}_{3}$ : Existe relação negativa entre a ambiguidade de papéis e o desempenho gerencial.

A partir das evidências supracitadas, nota-se que diversos estudos foram desenvolvidos sob a perspectiva comportamental da contabilidade, até mesmo com ênfase nos efeitos cognitivos (ambiguidade de papéis) da participação orçamentária no desempenho gerencial. Apesar disso, especificamente sobre a perspectiva teórica dos papéis, poucos estudos foram realizados sob esta temática no Brasil, o que torna oportuno a realização desta pesquisa.

\section{Método e procedimentos da pesquisa}

Esta pesquisa caracteriza-se como descritiva, realizada por meio de um levantamento dos dados e com abordagem quantitativa. $\mathrm{O}$ modelo teórico de análise aqui proposto é apresentado na figura 1.

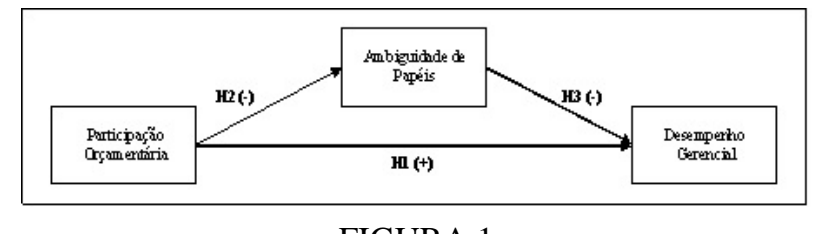

FIGURA 1

Modelo teórico de análise

Fonte: elaborado pelos autores.

\section{Procedimento de coleta dos dados}

Para coletar os dados, utilizou-se um questionário on-line, que garantia o anonimato dos participantes do estudo e da empresa em que atuam. Empregaram-se três variáveis para testar as hipóteses. A primeira parte do questionário abrangeu as percepções dos gestores sobre a participação orçamentária, a partir do instrumento de pesquisa desenvolvido por Milani (1975), que contém seis indicadores. A segunda parte compreendeu a variável ambiguidade de papéis, utilizando-se o instrumento desenvolvido por Rizzo et al. (1970), que compreende também seis indicadores. Para medir a variável de desempenho gerencial, foi usado o instrumento de Mahoney, Jerdee e Carroll $(1963 ; 1965)$, composto por nove itens. O instrumento de pesquisa utilizado para a coleta dos dados encontra-se em anexo.

Inicialmente os instrumentos empregados para a coleta de dados foram traduzidos do inglês para o português com o apoio de um profissional da língua inglesa, com conhecimentos avançados nos idiomas português e inglês. Posteriormente, foram escolhidas as traduções mais apropriadas para os instrumentos originais. Em seguida, os instrumentos foram comparados com os utilizados em pesquisas realizadas no Brasil na área contábil que aplicaram estes instrumentos (Palomino, 2013; Zonatto, 2014). Para medir a força de cada um dos indicadores, utilizou-se uma escala de medição Likert, composta por níveis de valor contínuo, ou seja, o grau de concordância varia entre (1) discordo totalmente e (7) concordo totalmente para as variáveis participação orçamentária e ambiguidade de papéis e (1) desempenho abaixo da média e (7) desempenho acima da média para o constructo de desempenho gerencial.

Depois que o instrumento foi finalizado, e antes de iniciar a coleta dos dados, foi realizado um pré-teste. Esta etapa desenvolveu-se com o auxílio de um professor pesquisador da área da contabilidade gerencial, Doutor em Ciências Contábeis e especialista nesta temática, que realizou análises da tradução, adaptações realizadas e as terminologias utilizadas na pesquisa orçamentária no Brasil, bem como de dois estudantes de doutorado em Ciências Contábeis e Administração. O instrumento também foi apreciado por dois gestores de empresas de grande porte do Brasil. Este processo permitiu a detecção de indicadores no questionário que, 
na visão dos revisores, não estavam claros, e na sequência foram corrigidos. Após uma segunda análise pelos profissionais citados, foram aceitos e considerados finalizados.

Igualmente, com vistas a identificar problemas no instrumento de pesquisa, foi feito um pré-teste estatístico e uma validação pelo teste de confiabilidade alfa de cronbach. Este pré-teste foi efetuado em uma amostra de 37 gestores com responsabilidade orçamentária, o que corresponde a $24 \%$ da amostra final analisada. Uma vez que as alterações solicitadas pelos profissionais foram concluídas e os indicadores dos constructos validados por meio do teste estatístico, a versão final do questionário foi enviada para os gestores foco do estudo.

A coleta dos dados foi realizada com o auxílio de pesquisadores do Centro de Estudos e Pesquisas em Administração (CEPA), órgão vinculado à Escola de Administração da Universidade Federal do Rio Grande do Sul (EA/UFRGS). O questionário foi aplicado por dois pesquisadores que atuam no CEPA, por meio de contato telefônico com a população objeto de estudo, no início do mês de setembro de 2015 com término no mês de outubro do mesmo ano. Estes pesquisadores receberam orientações sobre o objetivo, os constructos teóricos utilizados e o instrumento da pesquisa, com vistas a sanarem as eventuais dúvidas dos respondentes do estudo.

\section{População e amostra}

A população objeto deste estudo compreendeu os gestores com responsabilidades orçamentárias das maiores empresas brasileiras do ramo de exportação que possuem configuração orçamentária participativa. A amostra da pesquisa foi constituída por organizações industriais dos segmentos de alimentos, farmacêutico, químicos, madeira, móveis, siderurgia, usinagem, fundição, mineração, tecelagem, tecnologia e veículos. A escolha por essas organizações ocorreu de modo a investigar de forma abrangente o comportamento dos gestores brasileiros diante da participação orçamentária.

Justifica-se analisar gestores com responsabilidades orçamentárias que atuam nas maiores empresas com operações de exportação, pois esta escolha é convergente a critérios utilizados em pesquisas desenvolvidas sobre a temática central analisada (participação orçamentária), como por exemplo, a pesquisa de Zonatto (2014). Do mesmo modo, conforme Zonatto (2014, p. 163), as empresas de grande porte e com operações internacionais "oferecem um campo adequado de estudo para a investigação comportamental na contabilidade".

A definição da população desta pesquisa foi estabelecida a partir da identificação das maiores empresas em operações de exportação no país, sendo pesquisados os gestores com responsabilidade orçamentária que atuam em ao menos um dos seguintes departamentos: orçamento, controladoria, contabilidade, administração, comercial, financeiro e/ou produção. Delimitou-se ainda como critério de participação na pesquisa o número máximo de dois respondentes por empresa. Da listagem que contempla as 400 maiores empresas do ramo de exportação, foram contatadas 379 empresas. Após este contato, obteve-se a participação de 150 gestores com responsabilidades orçamentárias de 142 diferentes empresas.

\section{Procedimento de análise dos dados}

No que respeita aos procedimentos de análise dos dados, realizou-se a análise de trajetórias (path analysis) com vistas a validar o modelo teórico proposto. Contudo, antes desenvolveu-se a análise fatorial com o propósito de explicar o conjunto de variáveis de cada constructo. A carga fatorial para amostras de 150 respondentes deve ser maior que 0,45 para ser considerada significativa. $O$ teste de esfericidade de Bartlett indicou que as correlações são suficientes para continuar a análise dos dados e obteve-se uma significância de $<0,05$. Posteriormente, foi avaliada a confiabilidade dos constructos por meio do Alfa de Cronbach. Os resultados 
deste teste revelaram uma confiabilidade dos constructos acima de 0,60 , sendo considerados como adequados conforme à literatura (Hair Jr. et al., 2009).

$\mathrm{Na}$ sequência, realizou-se a análise de trajetórias que objetiva revelar a associação entre variáveis em diferentes efeitos, tanto diretos quanto indiretos, bem como a observação dessas variáveis em um conjunto de relações causais. Para tanto, desenvolveu-se inicialmente a análise da matriz de correlação dos constructos, seguido do coeficiente de correlação, que indica a força da associação entre as variáveis, que pode variar de -1 $a+1$ (Hair Jr. et al. 2009). Depois efetuou-se a avaliação do coeficiente de determinação $\left(R^{2}\right)$, que apresenta o poder de explicação do modelo e o teste de valores parciais $F($ ou $\mathrm{f}$ ) que verifica a contribuição de uma variável para o modelo. Para o teste de significância dos caminhos propostos utilizou-se $p$-value $<0,05$. Além disso, as trajetórias foram analisadas a partir do coeficiente beta padronizado, pois permite a comparação direta entre os coeficientes (Marôco, 2011).

\section{Descrição e análise dos resultados}

Primeiramente apresentam-se os resultados sobre os efeitos cognitivos da participação orçamentária. Seguidamente evidencia-se a discussão dos efeitos da participação orçamentária sobre o desempenho gerencial, por meio da ambiguidade de papéis.

\section{Efeitos cognitivos da participação orçamentária}

Nesta etapa da análise de trajetórias, estabeleceu-se como variável independente a participação orçamentária e como variável dependente a ambiguidade de papéis. Para a segunda análise, utilizou-se como variáveis independentes a participação orçamentária e ambiguidade de papéis e como variável dependente o desempenho gerencial. A tabela 1 apresenta os resultados destas relações.

TABELA 1

Sumário do modelo de avaliação dos efeitos cognitivos da Participação Orçamentária

\begin{tabular}{|l|c|c|c|c|c|}
\hline Variáveis dependentes & $\mathbf{R}$ & $\mathbf{R}^{\mathbf{2}}$ & $\begin{array}{c}\text { Erro } \\
\text { Padrão }\end{array}$ & $\mathbf{F}$ & $P$-value \\
\hline Ambiguidade de papéis & $-0,413$ & 0,171 & 2,24988 & 30,507 & 0,000 \\
\hline Desempenho gerencial & 0,387 & 0,159 & 3,32391 & 12,951 & 0,000 \\
\hline
\end{tabular}

Fonte: dados da pesquisa.

Na tabela 1, observa-se que a variável dependente ambiguidade de papéis apresenta um coeficiente de correlação $(\mathrm{R})$ negativo de $-0,413$. Nota-se que a variável ambiguidade de papéis consegue explicar $17,10 \%$ da participação orçamentária dos gestores nos processos orçamentários. A partir destes achados podemse constatar os benefícios para os gestores de participarem dos processos orçamentários, pois este processo esclarece a parcela do orçamento em que os subordinados estão envolvidos na configuração orçamentária, os tipos de raciocínios necessários para os superiores revisarem o orçamento, a frequência das discussões orçamentárias com o supervisor, a quantidade de influência que este possui no orçamento final da sua unidade e inclusive a importância da sua contribuição para a realização do orçamento (Milani, 1975). Tais benefícios da participação dos processos orçamentários apresentam reflexos na diminuição da ambiguidade de papéis dos subordinados, dado que assim terão maiores informações para desenvolverem suas tarefas.

Quanto à variável dependente desempenho gerencial, observa-se um coeficiente de determinação $\left(\mathrm{R}^{2}\right)$ de 0,159 , o que sugere que a participação orçamentária e a ambiguidade de papéis apresentam efeitos no desempenho dos gestores com responsabilidade orçamentária no trabalho. Este resultado evidencia o papel cognitivo da participação orçamentária, pois o processo de definição e envolvimento na elaboração do orçamento oferece aos subordinados a oportunidade de reunir, trocar e disseminar informaçóes importantes 
para o desenvolvimento de suas tarefas e proporciona, da mesma maneira, melhores tomadas de decisões e desempenho gerencial (Chong, 2002; Chong \& Chong, 2002).

$\mathrm{Na}$ figura 2 apresenta-se a análise de trajetórias dos efeitos cognitivos da participação orçamentária sobre o desempenho gerencial.

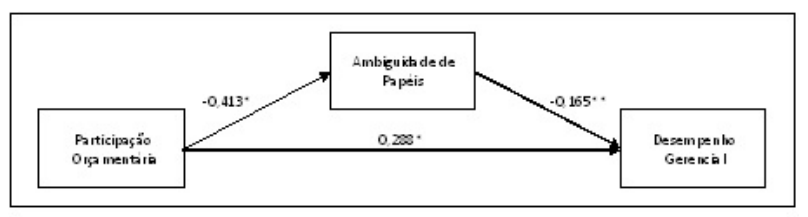

FIGURA 2

Coeficientes de trajetórias do modelo de análise dos efeitos cognitivos da Participação Orçamentária *A correlação é significativa no nível 1\%. ${ }^{* *}$ A correlação é significativa no nível 5\%. Fonte: dados da pesquisa.

A figura 2 evidencia os coeficientes encontrados na análise das trajetórias para a abordagem dos efeitos cognitivos da participação orçamentária e as relações propostas nesta pesquisa. Repara-se na figura 2 o efeito da participação orçamentária no desempenho gerencial e na ambiguidade de papéis. Denota-se igualmente, um efeito indireto entre a participação orçamentária e o desempenho gerencial, por meio da ambiguidade de papéis. Com vistas a um melhor detalhamento dos resultados obtidos, na Tabela 2 apresentam-se os coeficientes da regressão do modelo de análise dos efeitos cognitivos da participação orçamentária.

TABELA 2

Coeficientes da regressão do modelo de análise dos efeitos cognitivos da participação orçamentária

\begin{tabular}{|l|l|c|c|c|}
\hline \multicolumn{1}{|c|}{$\begin{array}{c}\text { Variável } \\
\text { dependente }\end{array}$} & \multicolumn{1}{|c|}{$\begin{array}{c}\text { Variável } \\
\text { independente }\end{array}$} & $\begin{array}{c}\text { Coeficiente de } \\
\text { trajetória }\end{array}$ & T-value & $P$-value \\
\hline $\begin{array}{l}\text { Ambiguidade de } \\
\text { Papéis }\end{array}$ & $\begin{array}{l}\text { Participação } \\
\text { orçamentária }\end{array}$ & $-0,413$ & $-5,523$ & 0,000 \\
\hline \multirow{2}{*}{$\begin{array}{l}\text { Desempenho } \\
\text { Gerencial }\end{array}$} & $\begin{array}{l}\text { Participação } \\
\text { orçamentária }\end{array}$ & 0,288 & 3,449 & 0,001 \\
\cline { 2 - 5 } & $\begin{array}{l}\text { Ambiguidade de } \\
\text { papéis }\end{array}$ & $-0,165$ & $-1,981$ & 0,05 \\
\hline
\end{tabular}

Fonte: dados da pesquisa.

Conforme a tabela 2, no que se refere ao efeito direto entre a participação orçamentária e o desempenho gerencial, os resultados revelaram uma relação positiva $(0,288)$. Estes achados permitem aceitar a hipótese de pesquisa $\mathbf{H}_{1}$ : existe relação positiva entre a participação orçamentária e o desempenho gerencial. Este resultado corrobora os achados dos estudos desenvolvidos por Zonatto e Lavarda (2013), Santos et al. (2014), Almasi et al. (2015), Hariyanti et al. (2015), Zonatto et al. (2019) e Jacomossi et al. (2019).

A partir desta evidência, é possível ratificar os argumentos de Milani (1975), pois o autor aborda a importância da participação dos subordinados no processo orçamentário, já que esta participação proporciona o compartilhamento de informações entre superior e seus subordinados, coordenação de atividades orçamentárias, motivação dos subordinados e promoção de atitudes e comportamentos positivos no trabalho (Milani, 1975; Shields \& Shields, 1998), o que reflete conjuntamente no grau em que os gestores orçamentários obtêm êxito na realização das atividades orçamentárias (Parker \& Kyj, 2006).

Francis-Gladney et al. (2004) também destacam que a participação orçamentária melhora o desempenho gerencial dos subordinados, porquanto estes estarão mais presentes no desenvolvimento do orçamento das suas unidades, o que desencadeia participações em reuniões orçamentárias, elaboração de relatórios orçamentários, fazendo com que haja mais comunicação entre as partes e desempenho no trabalho.

Zonatto e Lavarda (2013) encontraram evidências de uma relação positiva entre participação orçamentária e desempenho gerencial, em virtude de que o orçamento é considerado peça chave para a formulação de objetivos, metas e também é utilizado para avaliação de desempenho. Verifica-se a importância dos 
subordinados participarem dos processos orçamentários, em razão de apresentar reflexos positivos no desempenho (Almasi et al., 2015).

Tais resultados revelam similarmente, que a partir do momento em que são compartilhados os objetivos e metas orçamentárias por meio da participação orçamentária, este fato facilitará a alocação dos recursos e o desenvolvimento das atividades, o que impacta positivamente no desempenho gerencial (Zonatto et al., 2019). Nesse sentido, as empresas devem buscar o envolvimento e a influência dos gestores na definição das metas e objetivos orçamentários, vistos os seus impactos benéficos no desempenho destes (Macinati, Bozzi e Rizzo, 2016) nas atividades orçamentárias.

A participação orçamentária revelou um coeficiente de trajetória negativo $(-0,413)$ sobre a ambiguidade de papéis. Este achado possibilita suportar a segunda hipótese deste estudo, considerando que constatou-se que $\mathrm{H}_{2}$ existe relação negativa entre a participação orçamentária e a ambiguidade de papéis. Estas evidências se assemelham aos resultados dos estudos realizados por Chenhall e Brownell (1998), Chong e Bateman (2000), Chong (2002), Chong et al. (2006); Parker e Kyj (2006), Jermias e Yigit (2013) e Zonatto et al. (2019).

Estes resultados mostram a redução da ambiguidade de papéis devida ao efeito cognitivo observado a partir do compartilhamento de informações decorrentes da participação dos gestores nos processos orçamentários. Além disso, indicam que a participação orçamentária pode auxiliar os gestores com responsabilidades orçamentárias a entender as expectativas dos papéis, estabelecendo metas, tarefas e responsabilidades claras para cada posição ao elaborar o orçamento das suas unidades. Denota-se que os gestores com responsabilidades orçamentárias pesquisados possuem clareza das expectativas em relação aos seus papéis de trabalho (Chenhall \& Brownell, 1988; Chong \& Bateman, 2000; Chong et al., 2006), visto que a participação orçamentária possibilitou minimizar a ambiguidade de papéis existente no contexto orçamentário analisado.

Percebe-se a partir deste resultado que os gestores analisados possuem informações para desempenhar suas funções nas empresas pois, conforme Fisher (2001) e Palomino (2013), a ambiguidade de papéis é considerada uma tensão organizacional que pode ser provocada pela falta de informação para que os subordinados desenvolvam suas funções e dessa forma possuem incerteza sobre determinadas tarefas (Palomino \& Frezatti, 2016).

Nota-se que no cenário brasileiro a participação orçamentária está proporcionando aos indivíduos o envolvimento necessário para que a ambiguidade de papéis seja minimizada no ambiente de trabalho. Isto é positivo já que caso contrário, os subordinados poderiam se sentir insatisfeitos com as suas tarefas, o que consequentemente provocaria atitudes e comportamentos que fazem com que estes desenvolvam seu trabalho de maneira ineficaz diante da proposta orçamentária (Rizzo et al., 1970).

A participação orçamentária pode auxiliar no esclarecimento das tarefas, como realizá-las, a forma como o indivíduo será avaliado na organização e as consequências de atender ou não às expectativas dos superiores (Jermias \& Yigit, 2013), o que faz com que este participe ativamente do processo orçamentário compartilhando informações (Shields \& Shields, 1998). Assim minimiza-se a ambiguidade de papéis existente. Contudo, conforme Lin e Ling (2018), as organizações contemporâneas operam em um ambiente em que é quase impossível de se evitar a ambiguidade de papéis.

Por fim, a relação entre a ambiguidade de papéis e o desempenho gerencial resultou em um coeficiente de trajetória negativo $(-0,165)$. A partir deste resultado pode-se aceitar a hipótese $\mathbf{H}_{3}$, porquanto constatou-se que existe relação negativa entre a ambiguidade de papéis e o desempenho gerencial. Este achado está de acordo com os resultados encontrados por Chenhall e Brownell (1988), Tubre e Collins (2000), Fischer (2001), Chong (2002), Marginson e Ogden (2005), Parker e Kyj (2006), Chong et al. (2006), Wu e Norman (2006), Chong e Jonhson (2007), Burney e Widener (2007), Gilboa et al. (2008), Burkert et al. (2011), Jermias e Yigit (2013), Marginson et al. (2014), Beuren et al. (2017), Beuren et al. (2018) e Zonatto et al. (2019).

Este resultado comprova a importância de as empresas comunicarem especificações, informações e autoridade para a execução das tarefas pelos gestores, posto que a não existência destas afeta negativamente o desempenho gerencial (Beuren et al., 2017). Quando os gestores não possuem clareza sobre sua autoridade, 
objetivos, metas, responsabilidades, bem como explicações claras sobre suas tarefas, estas questões influenciam negativamente o desempenho destes no trabalho, nas atividades voltadas ao planejamento, investigação, coordenação, avaliação, supervisão, seleção, negociação e representação (Beuren et al., 2017).

Indica-se dessa maneira que a participação dos gestores com responsabilidades orçamentárias na elaboração do orçamento e o seu envolvimento ativo neste processo, contribui para a redução da ambiguidade de papéis e assim, melhora o desempenho gerencial (Chenhall \& Brownell, 1988; Chong et al., 2006; Zonatto et al., 2019). Portanto, níveis elevados de ambiguidade de papéis resultam em menor desempenho do indivíduo no trabalho e, por outro lado, baixos níveis de ambiguidade de papéis são suscetíveis de alavancar o desempenho gerencial (Chenhall \& Brownell, 1988; Parker \& Kyj, 2006; Beuren et al., 2017), sendo que os subordinados estarão interessados em esclarecer melhor as suas expectativas dos papéis que desempenham (Burkert et al., 2011) e compartilharão mais informações com seus superiores (Shields \& Shields, 1998; Parker \& Kyj, 2006; Chong \& Jonhson, 2007). Por conseguinte, terão informações mais claras sobre os seus deveres, responsabilidades do cargo e o comportamento esperado do executivo na função de controller (Palomino \& Frezatti, 2016).

Manifesta-se que a ambiguidade de papéis pode ser considerada um desafio nas empresas porque pode ser percebida pelos subordinados como uma ameaça no ambiente de trabalho, uma vez que estão acostumados com operações padronizadas (Gilboa et al., 2008). É nesse contexto que o papel dos gestores deve ser eficaz no sentido de planejar, estabelecer direções, organizar as tarefas e agir com outros fatores conjuntos para que os subordinados obtenham o desempenho gerencial que almejam no desenvolvimento de suas tarefas orçamentárias (Schermerhorn Jr. et al., 2007), o que em consequência pode minimizar a ambiguidade de papéis dos gestores e melhorar o seu desempenho gerencial no trabalho.

A partir das evidências encontradas neste estudo, verifica-se que a participação orçamentária oferece aos subordinados estrutura e clareza das tarefas que devem ser desenvolvidas (Marginson \& Ogden, 2005; Zonatto et al., 2019). Deste modo, a partir do momento em que a ambiguidade de papéis passa a ser minimizada pela participação orçamentária, haverá um maior compromisso dos indivíduos com as tarefas orçamentárias de suas unidades e isso refletirá no desempenho (Marginson \& Ogden, 2005).

Em síntese, as evidências achadas a partir do modelo teórico pesquisado sugerem que a participação orçamentária contribui para a redução da ambiguidade de papéis e a obtenção de melhor desempenho do gerente no trabalho, confirmando o papel cognitivo da participação orçamentária no desempenho gerencial. Esses resultados permitem concluir que a participação orçamentária apresenta efeitos cognitivos no desempenho dos indivíduos no trabalho, e ainda, destaca-se que a participação orçamentária apresenta efeitos diretos e indiretos no desempenho gerencial (Palomino \& Frezatti, 2016).

\section{Discussão dos resultados dos efeitos cognitivos da participação orçamentária sobre o desempenho gerencial}

A interação constante entre um indivíduo e outras pessoas nas organizações é denominada processo de socialização e é nesse processo que os indivíduos assumem papéis, posições e responsabilidades diante de um contexto (Palomino \& Frezatti, 2016). Nesse ambiente podem ocorrer ambiguidades relacionadas aos papéis dos indivíduos nas organizações. Nesta pesquisa observou-se que a participação orçamentária auxiliou para a diminuição desta ambiguidade e consequentemente melhorou o desempenho gerencial dos gestores com responsabilidades orçamentárias das maiores empresas brasileiras do ramo de exportação. Em concordância, a literatura tem mostrado que o nível de envolvimento dos gestores diante das metas traçadas tende a crescer quando estes são engajados no processo de desenvolvimento de açóes relacionadas ao contexto orçamentário (Jacomossi et al., 2019). 
De acordo com Zonatto et al. (2019), a participação orçamentária atua como um instrumento de gestão que possibilita reduzir a ambiguidade de papéis presente no contexto orçamentário. Para tanto, uma alternativa para que os indivíduos possam superar as restrições presentes no ambiente de trabalho está relacionada à necessidade de maior influência, bem como envolvimento e esforço para com a realização das atividades, de forma que o desempenho gerencial seja alcançado, mesmo em situações em que as condições de trabalho não sejam as mais adequadas.

Nesse sentido, a participação nos processos orçamentários da organização, oportuniza aos gestores compartilhar informações relevantes para o trabalho. Por consequência, torna-se possível o estabelecimento de previsões orçamentárias mais adequadas, o que qualifica o processo decisório dos gestores. A troca de informações permite ao gestor avaliar a quantidade de recursos disponíveis ou necessários para o desenvolvimento de suas atividades. Também oportuniza tomar conhecimento dos objetivos orçamentários da organização. Assim, nota-se que a participação orçamentária permite o esclarecimento das tarefas e melhora o desempenho dos indivíduos no trabalho, no que tange à realização dos seus papéis.

Da mesma forma, no modelo teórico analisado, a ambiguidade de papéis apresentou-se negativamente associada ao desempenho gerencial. Estes resultados revelam que a participação dos gestores nos processos orçamentários da organização reduz os níveis de ambiguidade de papéis e aumenta posteriormente o desempenho gerencial. Além disso, existem informações e especificações para a execução das tarefas na organização e os papéis dos gestores são claros (Beuren et al., 2017), o que possibilita elevar o desempenho dos gestores no trabalho. Neste contexto, observa-se que a participação orçamentária exerce um efeito cognitivo sobre o desempenho gerencial. Este efeito consiste na redução dos níveis de ambiguidade de papéis, resultando em melhor desempenho gerencial no trabalho dos gerentes com responsabilidade orçamentária.

Estes resultados reforçam as evidências sobre os efeitos dos papéis informativos da participação dos gestores dos processos orçamentários como um meio eficiente para minimizar os níveis de ambiguidade de papéis e consequentemente melhorar o desempenho destes em atividades orçamentárias. Deste modo, maiores níveis de participação orçamentária ocasionam maior confiança no ambiente de trabalho, o que favorece o compartilhamento de informações, a definição de metas e objetivos orçamentários, assim como o alcance de melhor desempenho nas atividades e inclusive o alcance de tais metas e objetivos (Zonatto et al., 2019).

Os achados desta pesquisa, no geral, foram consistentes com muitos esforços anteriores na literatura sobre participação orçamentária voltada para a tomada de decisão. Daqui se infere a relevância da análise de variáveis comportamentais no ambiente orçamentário. As evidências encontradas neste estudo também corroboram as de diversos estudos, como por exemplo, Zonatto (2014), Dani et al. (2017), Zonatto et al. (2019) e Jacomossi et al. (2019), pesquisas que também indicaram que poucos estudos têm sido realizados na área contábil com vistas a avaliar a relação entre a participação orçamentária e o desempenho gerencial, sob a visão de outras variáveis que podem auxiliar na explicação desta relação. Estas evidências estimularam a utilização da variável ambiguidade de papéis para o desenvolvimento deste estudo.

Conforme constatado no estudo de Derfuss (2009) e Dani et al. (2017), os achados revelaram que a participação orçamentária cria valor por meio da sua relação positiva com as variáveis relacionadas ao comportamento dos gestores (Birnberg et al., 2007). Ainda, os resultados sugerem que a participação orçamentária quando analisada e combinada com outras variáveis, como é o caso da ambiguidade de papéis, pode melhor predizer o desempenho gerencial dos gestores com responsabilidades orçamentárias (Derfuss, 2009; Dani et al., 2017; Zonatto et al., 2019; Jacomossi et al., 2019).

Em suma, a participação orçamentária influencia direta e indiretamente o desempenho dos gestores com responsabilidades orçamentárias, a partir da redução dos efeitos negativos da ambiguidade de papéis, o que ocorre impulsionado pela atuação dos líderes nas organizações, pois são estes as peças chave, para a criação de condições para um ambiente favorável à participação orçamentária e melhor desempenho gerencial. 


\section{Considerações finais}

Os resultados deste estudo fornecem uma visão do processo da influência do papel cognitivo da participação orçamentária no desempenho do trabalho dos subordinados. Especificamente, o papel cognitivo do orçamento participativo que possibilita reduzir os níveis de ambiguidade de papéis dos subordinados. Esse resultado é consistente com estudos empíricos desenvolvidos sobre esta temática. Os achados encontrados sugerem que dentre os benefícios da participação orçamentária está a diminuição da ambiguidade dos papéis e o aumento do desempenho gerencial. Em outras palavras, quando os gerentes possuem uma compreensão clara do papel que irão desenvolver, bem como suas tarefas a serem desenvolvidas, estes terão um melhor desempenho nas atividades orçamentárias.

Além disso, os achados constatados no presente estudo fornecem evidências empíricas adicionais para apoiar a robustez da teoria dos papéis e os resultados encontrados em estudos prévios que examinaram o papel cognitivo da participação orçamentária sobre o desempenho do trabalho dos subordinados. A análise da ambiguidade de papéis revela que esta é uma variável interveniente que deve ser considerada na relação entre a participação orçamentária e o desempenho gerencial. Estas evidências são consistentes com a visão de que a participação orçamentária auxilia na redução da ambiguidade dos papéis dos gestores com responsabilidade orçamentária, e que, a partir do momento em que esta se apresenta minimizada nas organizaçóes, passa a melhorar o desempenho dos indivíduos no trabalho.

Os resultados desta pesquisa apresentam algumas implicações práticas para o gerenciamento nas organizações no que respeita ao contexto orçamentário, visto que este estudo auxilia a gestão a entender melhor: o efeito do papel cognitivo da participação orçamentária na minimização da ambiguidade dos papéis. Em conjunto, os resultados encontrados ajudam os gestores a entender a importância de permitir que os subordinados se envolvam e participem ativamente do processo orçamentário, na medida em que isso apresenta reflexos positivos no desempenho destes no trabalho. Os achados também podem ser úteis para as organizações no que tange a sua avaliação da política de definições orçamentárias.

Diante dos evidentes efeitos cognitivos da participação orçamentária sobre o desempenho gerencial, tornase importante recomendar, conforme Palomino e Frezatti (2016), que as organizações desenvolvam novas políticas e práticas organizacionais que possibilitem aos gestores com responsabilidades orçamentárias lidar mais facilmente com a ambiguidade ao executar suas tarefas. Com esta recomendação espera-se que essas práticas diminuam a ambiguidade desses gestores e potencialize os níveis de desempenho destes no trabalho, permitindo que sejam mantidas estas condições.

Esta pesquisa contribui para a literatura existente sobre participação orçamentária no cenário brasileiro, pois localizaram-se poucas pesquisas no Brasil com a configuração aqui proposta, isto é, a inclusão da variável ambiguidade de papéis na análise da relação entre participação orçamentária e desempenho gerencial (Jacomossi et al., 2019; Zonatto et al., 2019). Outra contribuição é que por meio dos resultados pode-se constatar que a ambiguidade de papéis é uma importante variável mediadora na relação entre participação orçamentária e desempenho gerencial. Assim, permitir o envolvimento dos gestores com responsabilidades orçamentárias nos processos de definição de metas e objetivos orçamentários, bem como da elaboração do orçamento da sua unidade, desvela efeitos benéficos para as organizações.

Contudo, conforme pode-se constatar nesta pesquisa, os benefícios da participação no processo orçamentário só podem ser concretizados a partir do momento em que os gestores possuem uma melhor compreensão de seus papéis, quando percebem os objetivos e metas pelas quais são responsáveis e quando passam a ser comprometidos com esses objetivos e metas orçamentárias. Finalmente, este estudo lança luz sobre os efeitos cognitivos da participação orçamentária e as condições em que esta melhora o desempenho gerencial.

Uma das limitações do estudo é a utilização de uma amostra intencional e, portanto, os resultados demonstram em parte a realidade da amostra analisada em particular e as conclusões obtidas nesta pesquisa 
não podem ser generalizadas além da amostra considerada. Outra questão é o viés que pode ter ocorrido, em função da não consideração de variáveis de controle no modelo proposto. Sugere-se que sejam desenvolvidos outros estudos sobre a temática analisada, mas com outras variáveis cognitivas para a análise da influência indireta destas na relação entre a participação orçamentária e o desempenho gerencial.

\section{Referências}

Almasi, H., Palizdar, M., \& Parsian, H. (2015). Budgetary participation and managerial performance: The impact of information and environmental volatility. Management Science Letters, 5, 843-854. https://doi.org/10.5267/j. msl.2015.7.001

Beuren, I. M., Anzilago, M., \& Dal Vesco, D. G. (2018). Efeitos do uso diagnóstico e interativo de medidas de avaliação de desempenho na ambiguidade de papéis e no empoderamento psicológico. Cuadernos de Administración, 31(56), 55-80. https://doi.org/10.11144/Javeriana.cao.31-56.ucdim

Beuren, I. M., Roth, T. C., \& Anzilago, M. (2017). Efeitos da aplicação do princípio da controlabilidade no desempenho gerencial mediada pelo conflito e ambiguidade de papéis. Revista Universo Contábil, 13(3), 6-28. https://doi.o rg/10.4270/ruc.2017316

Birnberg, J. G. (2011). A proposed framework for behavioral accounting research. Behavioral Research in Accounting, 23(1), 1-43. https://doi.org/10.2308/bria.2011.23.1.1

Birnberg, J. G., Luft, J., \& Shields, M. D. (2007). Psychology theory in management accounting research. Handbooks of Management Accounting Research, I(1), 113-135. https://doi.org/10.1016/S1751-3243(06)01004-2

Burkert, M., Fischer, F., \& Schäffer, U. (2011). Application of the controllability principle and managerial performance: the role of role perceptions. Management Accounting Research, 22(3), 143-159. https://doi.org/10.1016/j.mar .2011.03.002

Burney, L., \& Widener, S. (2007). Strategic performance measurement systems, job-relevant information, and managerial behavioral responses-role stress and performance. Behavioral Research in Accounting, 19, 43-69. htt ps://doi.org/10.2308/bria.2007.19.1.43

Chenhall, R. H., \& Brownell, P. (1988). The effect of participative budgeting on job satisfaction and performance: Role ambiguity as an intervening variable. Accounting, Organizations and Society, 13(3), 225-233. https://doi. org/10.1016/0361-3682(88)90001-3

Chong, V. K. (2002). A note on testing a model of cognitive budgetary participation processes using a structural equation modeling approach. Advances in Accounting, 19, 27-51. https://doi.org/10.1016/S0882-6110(02)19 003-1

Chong, V. K., Eggleton, I. R., \& Leong, M. K. (2006). The multiple roles of participative budgeting on job performance. Advances in Accounting, 22, 67-95. https://doi.org/10.1016/S0882-6110(06)22004-2

Chong, V. K., \& Bateman, D. (2000). The effects of role stress on budgetary participation and job satisfactionperformance linkages: A test of two different models. Advances in Accounting Behavioral Research, 3, 91-118. $\mathrm{h}$ ttps://doi.org/10.1016/S1474-7979(00)03027-1

Chong, V. K., \& Chong, K. M. (2002). Budget goal commitment and informational effects of budget participation on performance: A structural equation modeling approach. Behavioral Research in Accounting, 14, 65-86. http s://doi.org/10.2308/bria.2002.14.1.65

Chong, V., \& Jonhson, D. M. (2007). Testing a model of the antecedents and consequences of budgetary participation on job performance. Accounting and Business Research, 37(1), 3-19. https://doi.org/10.1080/00014788.2007. 9730055

Covaleski, M. A., Evans III, J. H., Luft, J. L., \& Shields, M. D. (2003). Budgeting research: three theoretical perspectives and criteria for selective integration. Journal of Management Accounting Research, 15, 3-49. https://doi.org/10 $.2308 /$ jmar.2003.15.1.3 
Covaleski, M., Evans III, J. H., Luft, J., \& Shields, M. D. (2007). Budgeting research: three theoretical perspectives and criteria for selective integration. In: C. Chapman, A. Hopwood, \& M. Shields. Handbook of Management Accounting Research, (v. 2, pp. 587-624).

Dani, A. C., Zonatto, V.C. da. S., \& Diehl, C. A. (2017). Participação orçamentária e desempenho gerencial: uma metaanálise das relações encontradas em pesquisas desenvolvidas na área comportamental da contabilidade. Advances in Scientific and Applied Accounting, 10(1), 54-72. https://doi.org/10.14392/ASAA.2017100104

Derfuss, K. (2009). The relationship of budgetary participation and reliance on accounting performance measures with individual-level consequent variables: A meta-analysis. European Accounting Review, 18(2), 203-239. https://d oi.org/10.1080/09638180802652371

Derfuss, K. (2016). Reconsidering the participative budgeting-performance relation: A meta-analysis regarding the impact of level of analysis, sample selection, measurement, and industry influences. The British Accounting Review, 48(1), 17-37. https://doi.org/10.1016/j.bar.2015.07.001

Etemadi, H., Dilami, Z. D., Bazaz, M. S., \& Parameswaran, R. (2009). Culture, management accounting and managerial performance: Focus Iran. Advances in Accounting, Incorporating Advances in International Accounting, 25, 216-225. https://doi.org/10.1016/j.adiac.2009.08.005

Fichter, C. (2011). A research study of role conlict, role ambiguity, job satisfaction, and burnout among inancial advisors. The Journal of American Academy of Business, 16(2), 54-59. https://spiral.lynn.edu/etds/248

Fisher, R. T. (2001). Role stress, the type: A behavior pattern, and external auditor job satisfaction and performance. Behavioral Research in Accounting, 13(1), 143-170. https://doi.org/10.2308/bria.2001.13.1.143

Francis-Gladney, L., Little, H. T., Magner, N. R., \& Welker, R. B. (2004). Does Organization-Mandated Budgetary Involvement Enhance Managers' budgetary Communication with their Supervisor? Advances in Management Accounting, 13(1), 167-182. https://doi.org/10.1016/S1474-7871(04)13007-4

Frezatti, F, Relvas, T. R. S., Junqueira, E., Nascimento, A. R. do, \& Oyadomari, J. C. (2010). Críticas ao orçamento: problemas com o artefato ou a não utilização de uma abordagem abrangente de análise? Advances in Scientific and Applied Accounting, 3(2), 190-216. http://www.atena.org.br/revista/ojs-2.2.3-06/index.php/ASAA/articl e/view/1764

Gilboa, S., Shirom, A., Fried, Y., \& Cooper, C. L. (2008). A meta-analysis of work demand stressors and job performance: examining main and moderating effects. Personal Psychology, 61, 227-271. https://doi.org/10.11 11/j.1744-6570.2008.00113.x

Hair Jr., J. F., Black, W. C., Babin, B. J., Anderson, R. E., \& Tatham, R. L. (2009). Análise multivariada de dados. 6. ed. São Paulo: Bookman.

Hariyanti, W., Purnamasari, P., \& Lestira O, M. (2015). Pluriform motivation as antecedent and its relationships to budgeting participation and managerial performance (Empirical Study on Manufacturing Companies listed on Indonesian Stock Exchange). Procedia-Social and Behavioral Sciences, 211, 836-843. https://doi.org/10.1016/j .sbspro.2015.11.110

Jacomossi, F., Schlup, D., \& Zonatto, V. C. da. S. (2019). Efeitos da participação orçamentária na relação entre ambiguidade e conflito de papéis e o desempenho gerencial. Advances in Scientific \& Applied Accounting, 11(3), 391-409. http://dx.doi.org/10.14392/asaa.2018110302

Jermias, J., \& Yigit, F. (2013). Budgetary participation in Turkey: The effects of information asymmetry, goal commitment, and role ambiguity on job satisfaction and performance. Journal of International Accounting Research, 12(1), 29-54. https://doi.org/10.2308/jiar-50385

Kyj, L., \& Parker, R. J. (2008). Antecedents of budget participation: leadership style, information asymmetry, and evaluative use of budget. Abacus, 44(4), 423-442. https://doi.org/10.1111/j.1467-6281.2008.00270.x

Lin, M., \& Ling, Q. (2018). Is role stress always harmful? Differentiating role overload and role ambiguity in the challenge-hindrance stressors framework. Tourism Management, 68, 355-366. https://doi.org/10.1016/j.tour man.2018.04.007 
Lucena, W. G. L., Fernandes, M. S. A., \& Silva, J. D. G. da. (2011). A contabilidade comportamental e os efeitos cognitivos no processo decisório: uma amostra com operadores da contabilidade. Revista Universo Contábil, 7(3), 41-58. https://doi.org/10.4270/RUC.2011321

Macinati, M. S., Bozzi, S., \& Rizzo, M. G. (2016). Budgetary participation and performance: The mediating effects of medical managers' job engagement and self-efficacy. Health Policy, 120(9), 1017-1028. https://doi.org/10.101 6/j.healthpol.2016.08.005

Mahoney, T. A., Jerdee, T. H., \& Carroll, S. J. (1963). Development of Managerial Performance: A Research Approach. Cincinnati, OH: South-Western Publishing.

Mahoney, T. A., Jerdee, T. H., \& Carroll, S. J. (1965). The job(s) of management. Industrial Relations, 4(2), 97-110. h ttps://doi.org/10.1111/j.1468-232X.1965.tb00922.x.

Marginson, D., \& Ogden, S. (2005). Coping with ambiguity through the budget: The positive effects of budgetary targets on managers' budgeting behaviours. Accounting, Organizations and Society, 30(5), 435-456. https://doi .org/10.1016/j.aos.2004.05.004

Marginson, D., McAulay, L., Roush, M., \& Van Zijl, T. (2014). Examining a positive psychological role for performance measures. Management Accounting Research, 25(1), 63-75. https://doi.org/10.1016/j.mar.2013.10.002

Marôco, J. (2011). Análise estatística com utilização do SPSS. 5. ed. Lisboa: Sílabo.

Milani, K. (1975). The relationship of participation in budget-setting to industrial supervisor performance and attitudes: a field study. The Accounting Review, 50(2), 274-284. https://www.jstor.org/stable/244709

Palomino, M. N. (2013). Conflito, ambiguidade de função e satisfação no trabalho: percepções dos controllers brasileiros. Dissertação, Mestrado em Contabilidade. Universidade de São Paulo. São Paulo.

Palomino, M. N., \& Frezatti, F. (2016). Role conflict, role ambiguity and job satisfaction: Perceptions of the Brazilian controllers. Revista de Administração, 51(2), 165-181. http://dx.doi.org/10.5700/rausp1232.

Parker, R. J., \& Kyj, L. (2006). Vertical information sharing in the budgeting process. Accounting, Organizations and Society, 31, 27-45. https://doi.org/10.1016/j.aos.2004.07.005

Rizzo, J. R., House, R. J., \& Lirtzman, S. I. (1970). Role conflict and ambiguity in complex organizations. Administrative Science Quarterly, 15, 150-163. https://www.jstor.org/stable/2391486

Santos, A. C. dos., Lavarda, C. E. F., \& Marcello, I. E. (2014). Relação do conhecimento de gestão de custos e participação orçamentária com o desempenho dos gestores. Revista Brasileira de Gestão de Negócios, 16(50), 124-142. http://dx.doi.org/10.7819/rbgn.v16i50.1236

Schermerhorn Jr., J. R., Hunt, J. G., \& Osborn, R. N. (2007). Fundamentos de Comportamento Organizacional, 2. ed. Porto Alegre: Bookman.

Shields, J. F., \& Shields, M. D. (1998). Antecedents of participative budgeting. Accounting, Organizations and Society, 23(1), 49-76. https://doi.org/10.1016/S0361-3682(97)00014-7

Tubre, T. C., \& Collins, J. M. (2000). Jackson and Schuler (1985) Revisited: a meta-analysis of the relationships between role ambiguity, role conflict, and job performance. Journal of Management, 26(1), 155-169. https://d oi.org/10.1177/014920630002600104

Venkatesh, R., \& Blaskovich, J. (2012). The mediating effect of psychological capital on the budget participation-job performance relationship. Journal of Management Accounting Research, 24(1), 159-175. https://doi.org/10.23 08/jmar-50202

Wincent, J., \& Örtqvist, D. (2011). Examining positive performance implications of role stressors by the indirect influence of positive affect: A study of new business managers. Journal of Applied Social Psychology, 41(3), 699-727. https://doi.org/10.1111/j.1559-1816.2011.00733.x

Wu, L., \& Norman, I. J. (2006). An investigation of job satisfaction, organizational commitment and role conflict and ambiguity in a sample of Chinese undergraduate nursing students. Nurse Education Today, 26(4), 304-314. ht tps://doi.org/10.1016/j.nedt.2005.10.011 
Zonatto, V. C. S. (2014). Influência de fatores sociais cognitivos de capacidade, vontade e oportunidade sobre o desempenho gerencial nas atividades orçamentárias das maiores empresas exportadoras do Brasil. Tese, Doutorado em Ciências Contábeis e Administração, Universidade Regional de Blumenau, Blumenau.

Zonatto, V. C. S., \& Lavarda, C. E. F. (2013). Evidências dos efeitos da participação orçamentária na assimetria de informação, estresse ocupacional e desempenho no trabalho. Advances in Scientific and Applied Accounting, 6(1), 92-111. http://www.atena.org.br/revista/ojs-2.2.3-08/index.php/ASAA/article/viewFile/1765/1652

Zonatto, V. C. S., Weber, A., \& Nascimento, J. C. (2019). Efeitos da participação orçamentária na assimetria informacional, estresse ocupacional e desempenho gerencial. Revista de Administração Contemporânea, 23(1), 67-91. http://doi.org/10.1590/1982-7849rac2019170327

\section{ANEXO}

\section{Bloco 1 - Participação Orçamentária}

As afirmativas abaixo estão relacionadas aos gestores e sua "participação no processo orçamentário" da empresa em que atua. Por gentileza indicar até qual ponto você discorda ou concorda com as afirmações apresentadas a seguir. $\mathrm{O}$ grau de concordância varia entre (1) discordo totalmente e (7) concordo totalmente.

\begin{tabular}{|c|c|c|c|c|c|c|c|c|}
\hline \multirow{2}{*}{ N. } & \multirow{2}{*}{ Afirmativas sobre Participação Orçamentária } & \multicolumn{2}{|c|}{$\begin{array}{l}\text { Discordo } \\
\text { Totalmente }\end{array}$} & \multicolumn{5}{|c|}{$\longleftrightarrow \begin{array}{c}\text { Concordo } \\
\text { Totalmente }\end{array}$} \\
\hline & & 1 & 2 & 3 & 4 & 5 & 6 & 7 \\
\hline PO_01 & $\begin{array}{l}\text { Estou totalmente envolvido na elaboração do orçamento } \\
\text { da minha unidade. }\end{array}$ & & & & & & & \\
\hline PO_02 & $\begin{array}{l}\text { Meu superior fornece amplas informações quando o } \\
\text { orçamento da minha unidade é revisado. }\end{array}$ & & & & & & & \\
\hline PO_03 & $\begin{array}{l}\text { Na elaboração do orçamento da minha unidade tenho } \\
\text { autonomia para emitir opinião ou apresentar sugestôes } \\
\text { sobre o orçamento para meu superior, mesmo sem ser } \\
\text { perguntado. }\end{array}$ & & & & & & & \\
\hline PO_04 & $\begin{array}{l}\text { Possuo grande influência no orçamento final de minha } \\
\text { unidade. }\end{array}$ & & & & & & & \\
\hline PO_05 & $\begin{array}{l}\text { Acredito ser grande a minha contribuição para o processo } \\
\text { orçamentário em minha unidade. }\end{array}$ & & & & & & & \\
\hline PO_06 & $\begin{array}{l}\text { Ao definir o orçamento da minha unidade sou procurado } \\
\text { com frequência pelo meu superior para trocar } \\
\text { informações, emitir opiniōes e sugestões sobre as } \\
\text { previsões realizadas. }\end{array}$ & & & & & & & \\
\hline
\end{tabular}

\section{Bloco 2 - Ambiguidade de Papéis}

As afirmativas abaixo estão relacionadas ao "papel que o gestor assume" na empresa em que atua. Por gentileza indicar até qual ponto você discorda ou concorda com as afirmações apresentadas a seguir. $\mathrm{O}$ grau de concordância varia entre (1) discordo totalmente e (7) concordo totalmente.

\begin{tabular}{|c|c|c|c|c|c|c|c|c|}
\hline \multirow[t]{2}{*}{ N. } & \multirow{2}{*}{ Afirmativas sobre Ambiguidade de Papéis } & \multicolumn{5}{|c|}{$\begin{array}{l}\text { Discordo } \\
\text { Totalmente }\end{array}$} & \multicolumn{2}{|c|}{$\begin{array}{r}\text { Concordo } \\
\text { Totalmente }\end{array}$} \\
\hline & & 1 & 2 & 3 & 4 & 5 & 6 & 7 \\
\hline AP_01 & $\begin{array}{l}\text { Estou ciente da autoridade que tenho para a tomada de } \\
\text { decisões na minha unidade. }\end{array}$ & & & & & & & \\
\hline AP_02 & $\begin{array}{l}\text { Eu tenho metas planejadas e objetivos claros para a } \\
\text { execução das minhas tarefas. }\end{array}$ & & & & & & & \\
\hline AP_03 & $\begin{array}{l}\text { Meu tempo está distribuído de forma adequada para } \\
\text { atender diferentes tarefas. }\end{array}$ & & & & & & & \\
\hline AP_04 & Eu sei quais são as minhas responsabilidades. & & & & & & & \\
\hline AP_05 & Eu sei exatamente o que é esperado de mim. & & & & & & & \\
\hline AP_06 & As explicações sobre o que devo fazer são claras. & & & & & & & \\
\hline
\end{tabular}




\section{Bloco 3 - Desempenho Gerencial}

As afirmativas abaixo estão relacionadas à identificação do "desempenho gerencial nas atividades de trabalho" dos gerentes participantes da pesquisa. Por favor, auto avalie seu desempenho comparativamente ao de seus colegas, com base em cada uma das atividades (tarefas) especificadas a seguir. A escala de auto avaliação varia entre [1] desempenho abaixo da média [7] desempenho acima da média.

\begin{tabular}{|c|c|c|c|c|c|c|c|c|}
\hline \multirow{2}{*}{ N. } & \multirow{2}{*}{ Afirmativas sobre Desempenho Gerencial } & \multicolumn{2}{|c|}{$\begin{array}{l}\text { Desempenho } \\
\begin{array}{l}\text { Abaixo } \\
\text { Média }\end{array}\end{array}$} & \multicolumn{3}{|c|}{4} & \multicolumn{2}{|c|}{$\begin{array}{r}\begin{array}{r}\text { Desempenho } \\
\text { Acima da } \\
\text { Média }\end{array} \\
\end{array}$} \\
\hline & & 1 & 2 & 3 & 4 & 5 & 6 & 7 \\
\hline DG_01 & $\begin{array}{l}\text { Determinar metas, políticas e linhas de ação (por } \\
\text { exemplo, agendamento de trabalho, orçamento, } \\
\text { programação) }\end{array}$ & & & & & & & \\
\hline DG_02 & $\begin{array}{l}\text { Coleta e preparação de informações, geralmente sob a } \\
\text { forma de registros, relatório e contas (por exemplo, } \\
\text { medir a produção, manutenção de registros, análise de } \\
\text { trabalho) }\end{array}$ & & & & & & & \\
\hline DG_03 & $\begin{array}{l}\text { Trocar informações com as pessoas na organização que } \\
\text { não sejam seus subordinados, a fim de se relacionar e } \\
\text { ajustar programas (por exemplo, expedição, ligação com } \\
\text { outros gestores, organizando reuniões) }\end{array}$ & & & & & & & \\
\hline DG_04 & $\begin{array}{l}\text { Avaliar e apreciar as propostas ou relatos / desempenho } \\
\text { observado (por exemplo, avaliações de funcionários, a } \\
\text { julgar registros de saída, inspeção de produtos) }\end{array}$ & & & & & & & \\
\hline DG_05 & $\begin{array}{l}\text { Orientar, liderar, o desenvolvimento de seus } \\
\text { subordinados }\end{array}$ & & & & & & & \\
\hline DG_06 & $\begin{array}{l}\text { Manter a força de trabalho de sua unidade (por exemplo, } \\
\text { seleção e promoção de empregados) }\end{array}$ & & & & & & & \\
\hline DG_07 & $\begin{array}{l}\text { Adquirir, alienar, ou contratar bens e serviços (por } \\
\text { exemplo, negociações fiscais, contratação de } \\
\text { fornecedores, negociação coletiva, publicidade). }\end{array}$ & & & & & & & \\
\hline DG_08 & $\begin{array}{l}\text { Alavancar os interesses gerais da minha organização por } \\
\text { meio de palestras, consultas, ou contato com outras } \\
\text { pessoas fora da organização. }\end{array}$ & & & & & & & \\
\hline DG_09 & Como você avalia o seu desempenho global? & & & & & & & \\
\hline
\end{tabular}

\section{Notas}

* Artigo de pesquisa científica.Este artigo é resultado de uma pesquisa científica e tecnológica apoiada pelo CNPq. Artigo relacionado aos Projetos de Pesquisa Processos: 472195/2014-0 e 422179/2018-3.

\section{Licencia Creative Commons CC BY 4.0}

Para citar este artigo: Guidini, A. A., Zonatto, V. C. S., Degenhart, L., \& Schlup, D. (2020). Evidências da relação entre participação orçamentária, ambiguidade de papéis e o desempenho gerencial. Cuadernos de Contabilidad, 21. https://doi.org/10.11144/Javeriana.cc21.erpo 